\title{
The Application of Recurrence Quantification Analysis in Detection of Abrupt Climate Change
}

\author{
Wen Zhang, ${ }^{1,2}$ Guoling Feng, ${ }^{3,4}$ and Qunqun Liu ${ }^{3,5}$ \\ ${ }^{1}$ College of Atmospheric Sciences, Lanzhou University, Lanzhou 730000, China \\ ${ }^{2}$ Tongda College, Nanjing University of Posts \& Telecommunications, Nanjing 210003, China \\ ${ }^{3}$ National Climate Center, China Meteorological Administration, Beijing 100081, China \\ ${ }^{4}$ College of Physical Science \& Technology, Yangzhou University, Yangzhou 225002, China \\ ${ }^{5}$ College of Math \& Statistics, Nanjing University of Information Science \& Technology, Nanjing 210044, China
}

Correspondence should be addressed to Wen Zhang; zhangwen@njupt.edu.cn

Received 25 December 2015; Accepted 24 April 2016

Academic Editor: Yi Wang

Copyright (C) 2016 Wen Zhang et al. This is an open access article distributed under the Creative Commons Attribution License, which permits unrestricted use, distribution, and reproduction in any medium, provided the original work is properly cited.

This paper explores the possible application of recurrence quantification analysis in the detection of abrupt change of the dynamic structure of the climate system. It is discovered in the recurrence quantification analysis of the typical chaotic system-logistic model that the method may well distinguish the state of logistic model with different parameters, demonstrating its potential value in identifying the dynamic change of the system. When recurrence quantification analysis is later applied to the detection of abrupt change of average daily precipitation of all regions in China, the result indicates that the abrupt change of the dynamic structure corresponding to the precipitation of China in recent 50 years occurred in the late 1970s and the early 1980s. It is in agreement with the Chinese commonly recognized years of abruption; therefore the effectiveness is further demonstrated regarding the recognition of complexity of dynamical system.

\section{Introduction}

It is well known that climate system is a nonlinear and nonstationary dynamic system, the change of which may encounter discontinuity, and such discontinuity is generally called the abrupt climate change. The study of the phenomenon and theory of the abrupt climate change is an emerging field in the modern climatology and has been attracting more and more attention. Conducting the quantitative analysis of historical climate materials by various detection technologies of the abrupt change to determine the occurrence time of abrupt climate change and further find the mechanism of abrupt change by material diagnosis or numerical simulation is very significant for the accurate forecasting of climate in the future.

Abrupt climate change may be roughly divided into two major types: abrupt change of variables of the climate state in the statistical significance and change of the dynamic structure of the climate system. The first type exists in various climate observation materials and involves multiple time scales. Such abrupt change is not necessarily related to the change of the dynamic equation of the climate system but is a self-adaption process of the climate system, such as the conversion between cold and warm phases. The first type is mainly judged by whether there is obvious change of statistical features of variables of the climate state in the statistical significance, such as the mean value, variance, frequency distribution, and change trend [1]. Methods involved include sliding $t$-test, Crammer Method, Yamamoto Method, Mann-Kendall Method, Wavelet Analysis, and Heuristic Segmentation Algorithm [2-6]. The second type, that is, the detection of abrupt change of the dynamic structure, is an emerging field in recent years, such as conditional entropy [7] and approximate entropy method [8]. Livina et al. [9-11] have put forward many new detection methods of the abrupt change of time series on the basis of the long-term memory of time series. These methods are quite useful for the detection of abrupt change of the structure of climate dynamics.

Although great achievements have been made in detection methods of the abrupt climate change, no method is perfect. For example, the detection technology of abrupt change based on the long-term memory of time series and the method of power law tail exponent both require the fractal 
feature of time series. The traditional detection technology of abrupt climate change depending on the change of statistical features of variables of the climate state is also quite limited. On the one hand, the technology needs a large amount of samples and many methods require the time series to meet the normal distributional assumption; on the other hand, it is difficult to detect the abrupt change of the dynamic structure of the system by using those methods. Therefore, developing different detection technologies of the abrupt change contains quite important scientific significance and value of application.

The studies related to the interaction between time and space is involved in various fields of life, such as zoology and botany $[12,13]$. In the process of research, the recurrence plot method, firstly proposed by Eckmann et al. [14] in 1987, is a nonlinear technology to observe the features of the system state in the phase space. Recurrence plot method may expose some future knowledge about time series visually through the graph, such as the similarity contained in time series and the predictability of information. To quantitatively describe the physical significance exposed by the recurrence plot, Webber Jr. et al. [15-18] have proposed the recurrence quantification analysis, which can measure the complexity of time series effectively, assess, and classify the space recurrence graph of each time to determine differences and similarities of the dynamic structure, so as to detect the abrupt change of structure of the climate dynamics by the evolution of space recurrence graph. This method is advanced in the low requirement on the quantity of samples in the computation and strong noise resistance. As a result, this method has been widely applied to medical science [19], hydromechanics [20], and diagnosis of faults of the mechanical equipment [21-23].

Even if the recurrence quantification analysis has been widely applied to many disciplines and fields, it is seldom applied to climatology and almost has not been applied to the detection of abrupt climate change. Therefore, this paper studies the feasibility and effectiveness of recurrence quantification analysis in the detection of the abrupt change of the dynamic structure of time series. In the beginning, this paper verifies the effectiveness of recurrence quantification analysis in identifying the complexity of structure of the dynamic system by the classical chaotic model-logistic model and then applies the analysis to the detection of abrupt climate change of daily precipitation materials of all stations of China. The result indicates that recurrence quantification analysis is able to distinguish and identify well the dynamic nature of the system, and its application to the average daily precipitation materials in areas of China further proves its practicability and reliability.

\section{Introduction to Methods}

2.1. Recurrence Plot. Regarding one-dimensional original time series $\left\{x_{1}, x_{2}, \ldots, x_{n}\right\}$, in the Takens Embedding Theorem, when the embedding dimension is $m$ and the delay time is $\tau$, the vector of phase space after the reconstruction is

$$
X_{i}=\left\{x_{\mathrm{i}}, x_{i+\tau}, \ldots, x_{i+(m-1) \tau}\right\}, \quad i=1,2, \ldots, N
$$

and in the formula, $N=n-(m-1) \tau$. Defining the distance between any two vectors in the reconstructed phase space $d_{i, j}=\left\|X_{i}-X_{j}\right\|$ and selecting $r$ as the threshold value, it may result in the recurrence matrix $\left\{R_{i j}, i=1,2, \ldots, N, j=\right.$ $1,2, \ldots, N\}$, in which $R_{i j}$ is the step function and may be expressed as $R_{i j}=$ Heaviside $\left(r-d_{i j}\right)$. The expression of Heaviside $(\Theta)$ is

$$
\text { Heaviside }(x)= \begin{cases}1 & x \geq 0 \\ 0 & x<0\end{cases}
$$

When $R_{i j}=1$, depicting points at $(i, j)$ in the $N \times N$ twodimensional coordinates and the result is the recurrence plot.

2.2. Selection of Parameters. The selection of three parameters, namely, threshold value $r$, embedding dimension $m$, and delay time $\tau$, requires certain technique. In view of existing experience, the selection of parameters may adopt the following principle: threshold value $r$ is generally $0.15 \sim$ 0.2 times of the standard deviation of the series, and this paper selects 0.18 ; embedding dimension $m$ may be obtained according to the correlation dimension; delay time $\tau$ may be determined by the autocorrelation function method. As the selected length of sliding window in the time series is fixed in the detection of abrupt change, this paper selects the fixed embedding dimension and delay time for the convenience of comparing the complexity of subsequences with the same length; that is, $m=3$ and $\tau=5$.

2.3. Recurrence Quantification Analysis. To quantify the recurrence of the system demonstrated in the recurrence plot, Webber Jr. et al. have proposed some parameters of the recurrence quantification analysis, such as recurrence rate (RR), determinism (DET), and recurrence entropy. These parameters describe features of the recurrence plot from difference perspectives. This study only selects two parameters, $\mathrm{RR}$ and DET, to describe features of the recurrence plot.

$\mathrm{RR}$ is percentage between recurrence points in the recurrence plan and total points of the recurrence plan; that is,

$$
\mathrm{RR}=\frac{1}{N^{2}} \sum_{i, j=1}^{N} R_{i j} .
$$

DET is percentage between recurrence points forming diagonal segments and all recurrence points; that is,

$$
\mathrm{DET}=\frac{\sum_{l=l_{\min }}^{N-1} l \cdot p(l)}{\sum_{i, j=1}^{N} R_{i j}},
$$

and in the formula, $p(l)$ is quantity of segments in the length of $l$ and it is only counted when the length of diagonal segments is longer than the set lower limit $l_{\min } . l_{\min }$ is generally an integer not smaller than 2 and too large $l_{\min }$ may deteriorate the indicative significance of DET. DET will distinguish isolated recurrence points from recurrence points forming diagonal segments in the recurrence plot. The clearer the lines along the principal diagonal in the recurrence plot are, the stronger the determinism of the system is. 


\section{Test of Ideal Value}

For the purpose of verifying the identification of different dynamic systems by the recurrence plot method, this paper analyzes three groups of signals, namely, periodic signals, random signals, and chaotic signals in the beginning by the recurrence plot method. Each group of time series includes 1,000 samples. Periodic signals come from the periodic function below: $x(i)=\cos (2 \pi i / 100)$; random signals are generated by the random function. Chaotic series comes from the classical logistic model expressed as $x(i+1)=$ $u *(1-x(i))$, in which $u$ is 3.9 and integration step is 1 and 1,000 steps iterated initially are abandoned to eliminate the influence brought by the initial value. As the evolution of recurrence plot of chaotic signals and random signals is more complicated than that of periodic signals and it contains certain self-similarity, only a part of the recurrence plot is selected in Figures 1(b) and 1(c) to demonstrate its features more clearly.

It can be seen from Figure 1 that the graph of recurrence plot reflects the trend and discipline that the track of phase space changes along with the time. Recurrence plots corresponding to series with different dynamic structures are different from each other obviously. The recurrence plot of periodic signals contains strong periodicity that a large number of points form segments parallel with the principal diagonal; points on the recurrence plot of chaotic signals are denser than those on the recurrence plot of random signals. Besides, points on the recurrence plot of chaotic signals are not isolated and form a large amount of segments parallel with or vertical to the principal diagonal, but segments parallel with the principal diagonal are short and few, indicating that the long-term predictability of chaotic series is weaker and it may only be used for the short-term prediction mainly due to the sensitive dependence of chaotic series on initial conditions. Distribution of points on the recurrence plot of random signals is quite random, sparse, and irregular, indicating that random signals are unpredictable at all. As a result, recurrence plots corresponding to different dynamic systems are different from each other obviously and the result proves that the analysis of recurrence plot may distinguish different dynamic structures.

With respect to systems with the same dynamic structure, will the recurrence plot change obviously when any parameter or boundary condition is changed? To answer this question, we conduct the numerical experiment by logistic models. Existing studies have proved that in the logistic model when $u$ is $3 \sim 3.57$, the system will experience the period-doubling bifurcation, and when it is $3.57 \sim 4$, the system will enter the chaotic region and some parts in the chaotic region include special transparent zonal regions.

In view of different dynamic features of the logistic model within different parameter intervals, we investigate the identification capability of the system state by the recurrence plot when $u$ is $3.5,3.6,3.8$, and 3.85, respectively. Figure 2 demonstrates the recurrence plot of different states of the logistic model when the parameter is different. When $u=3.5$, the logistic model is a periodic function; when $u=3.6$, the system begins to become chaotic; when $u=3.8$, the logistic
TABLE 1: RR and DET corresponding to series of logistic models with different parameters.

\begin{tabular}{lcccc}
\hline Parameter $u$ & 3.50 & 3.60 & 3.8 & 3.85 \\
\hline RR & 0.250 & $4.66 E-02$ & $5.55 E-03$ & 0.333 \\
DET & 0.996 & 0.723 & 0.589 & 0.997 \\
\hline
\end{tabular}

model demonstrates typical chaotic behaviors; and when $u=3.85$, the system corresponds to the "transparent" zonal region in the chaotic region, which includes the multipoint periodic structure.

It can be seen from Figure 2 that the spatial difference between recurrence plots corresponding to different parameters is quite large, indicating that the recurrence plot can reflect the complexity of the system effectively. When the system demonstrates periodic behaviors, a large number of points form segments parallel with the principal diagonal; compared with periodic signals, chaotic system contains less segments parallel to the diagonal and the more chaotic it is, the more sparse the segments parallel with the diagonal are. As mentioned above, when $u=3.85$, the chaotic system corresponds to the "transparent" window in the phase space and the system demonstrates certain pseudoperiodic behaviors in the region, which is quite different from the recurrence plot when $u$ is 3.6 and 3.8. It is easy to find from Figure 2(d) that the recurrence plot seems like the punctuate distribution of the periodic behavior but the aggregation degree is sparser than that of the periodic behavior when compared with Figure 2(a), indicating that the recurrence plot may demonstrate such pseudoperiodic behaviors well.

The analysis result above is based on the subjective personal judgment. We have computed RR and DET of recurrence plots with different parameters for the quantitative analysis of the recurrence plot, and the result is listed in Table 1. It can be seen from Table 1 that, with respect to dynamic structures corresponding to different parameters, their states are different and their RR and DET change within different regions, indicating that the recurrence quantification analysis can well describe the complexity of the dynamic system. It can also be seen from the table that, with respect to different parameters, the difference of $R R$ is more obvious than that of DET.

The test above proves that the recurrence quantification analysis method can identify different dynamic structures, so we could discuss the application of recurrence quantification analysis method in the actual observation materials to inspect whether it may identify the abrupt change of dynamic structure of the climate system effectively. Besides, RR is more obvious towards different dynamic structures than DET, so we adopt RR in the following application for the purpose of comparison.

\section{Analysis of Precipitation of All Stations of China}

In order to inspect the application of the recurrence quantification analysis in the actual observation materials, we apply 


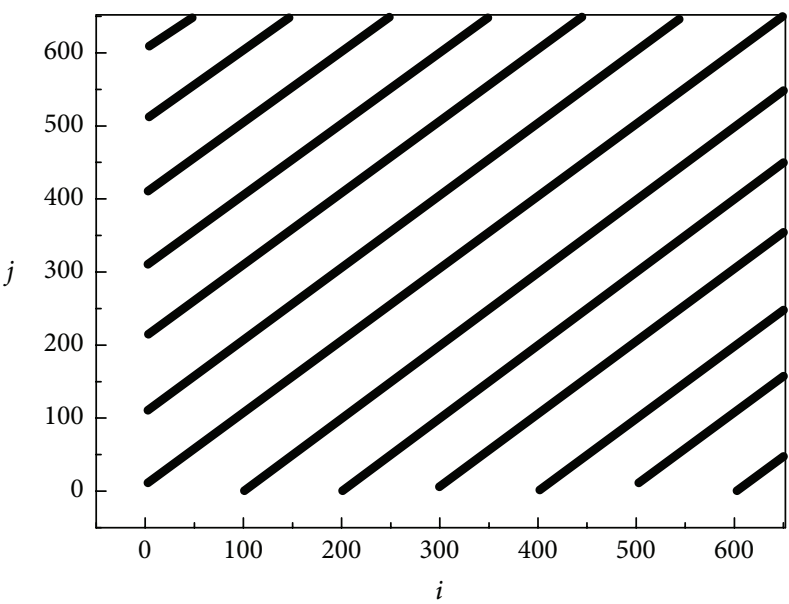

(a)

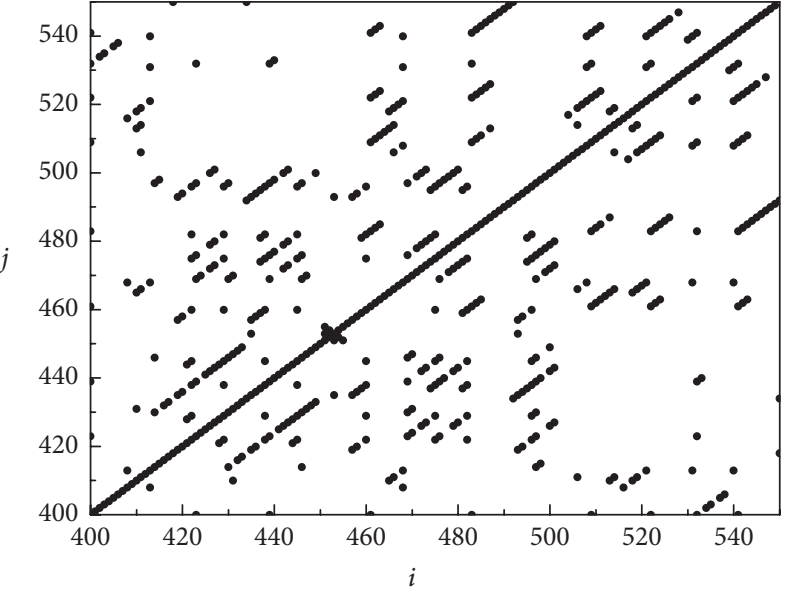

(b)

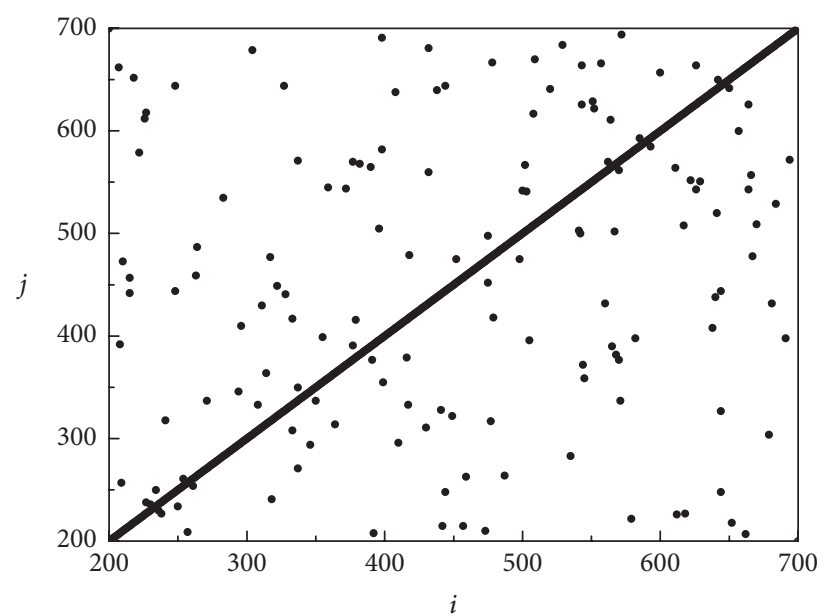

(c)

FIGURE 1: Recurrence plot of different dynamic systems: (a) periodic, (b) chaotic, and (c) random.

the recurrence quantification analysis method to the daily precipitation materials of 614 stations of China from 1960 to 2010, altogether 51 years. Calculate the annual RR corresponding to the daily precipitation of respective stations in China and compile the RR distribution charts corresponding to the precipitation of each observation station. It can be viewed generally that the features of spatial distribution of the RR corresponding to the daily precipitation materials of all stations remain basically unchanged over the past 50 years in China. They demonstrate that the dynamical system controlling the precipitation in China remains unchanged. It is discovered in the further analysis that the spatial distribution features of the annual RR corresponding to the daily precipitation of each station are similar to the characteristics of the distribution of climate types in China, which demonstrates the effectiveness and reliability of the RR in recognition of complexity of dynamical system. Based on the above research, eight climate zones are generally divided in China, shown in Figure 3. They are (a) south China, (b) the middle and lower reaches of the Yangtze River, (c) north China, (d) northeast China, (e) the east of northwest China, (f) the west of northwest China, (g) Tibet, and (h) southwest China. Calculate, respectively, the average annual RR of daily precipitation in each zone. The annual changing curve of $R R$ in each zone is shown in Figure 4.

It can be seen from Figure 4 that the RR of average annual precipitation of areas in China has the feature of the increase from the east to the west and from the south to the north. It indicates the extent of complexity corresponding to each region has the features of higher complexity in the west and the north and lower complexity in the east and the south, and the changing feature of annual average precipitation varies from region to region. The abrupt change of the average value takes place in some regions; therefore, in the paper $t$-test is applied to the examination of annual RR sequence of each region. Except the three regions of the middle and lower reaches of the Yangtze River, south China, and the southwest China, the RR of precipitation sequence corresponding to the five remaining regions demonstrated obvious abrupt changes (probability-confidence 99\%) in the late 1970s and the early 1980s, and the RR after abrupt changes is only around onethird of the RR before. The years when those above abrupt 


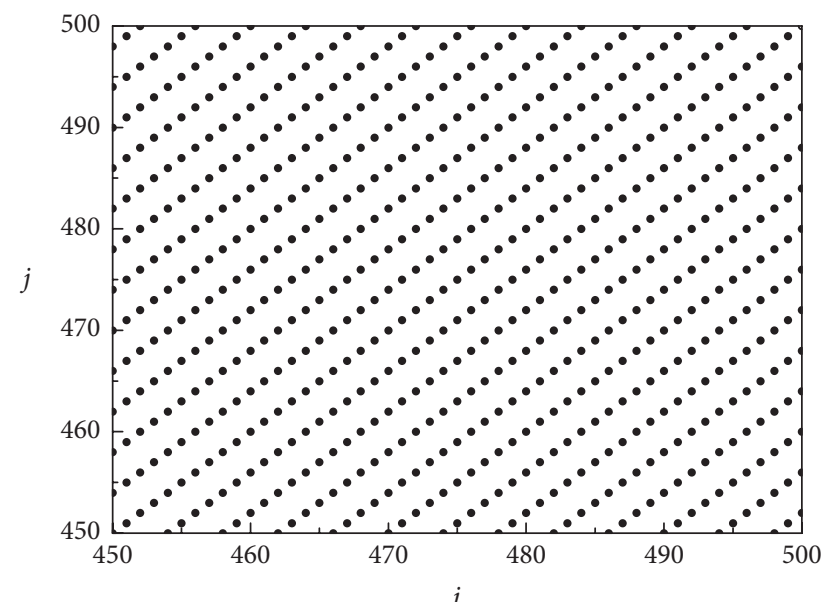

(a)

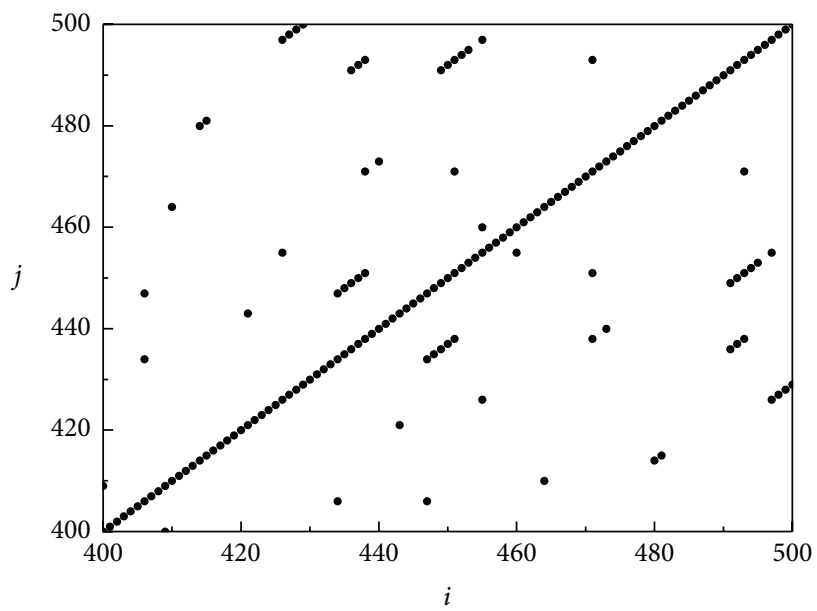

(c)

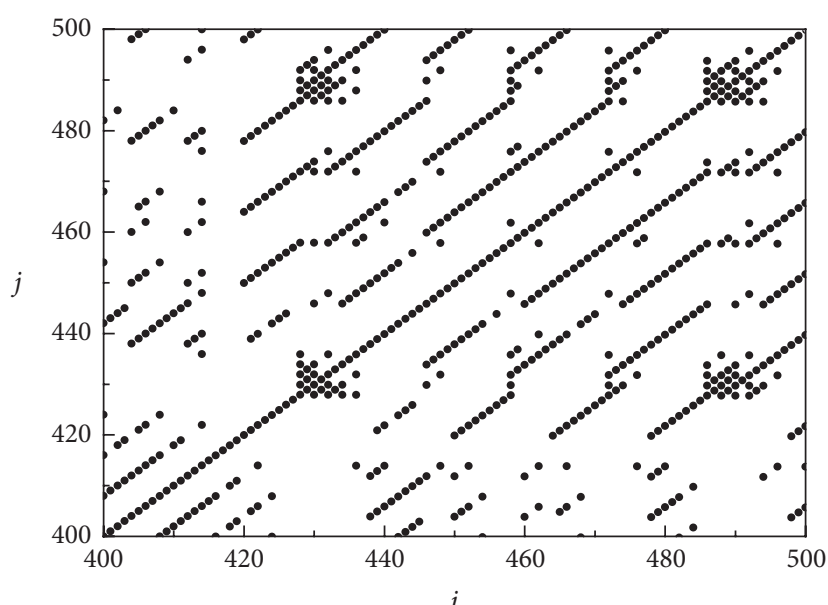

(b)

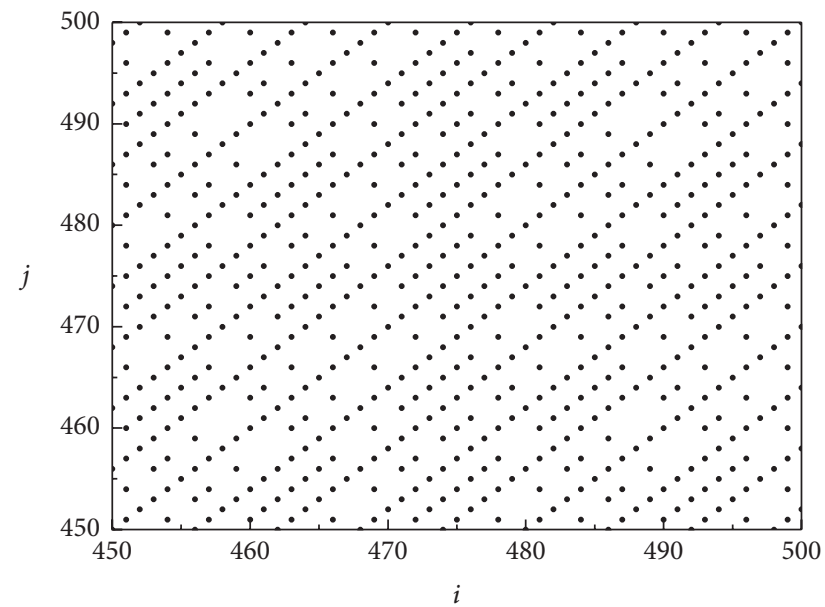

(d)

FIGURE 2: Recurrence plots corresponding to logical models with different parameters: (a) $u=3.5$; (b) $u=3.6$; (c) $u=3.8$; (d) $u=3.85$.

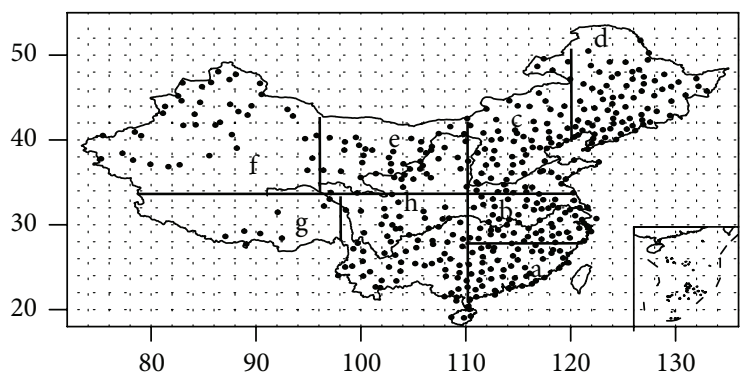

FIGURE 3: The map of climate regions in China.

changes took place are in agreement with those commonly recognized in China; therefore the effectiveness is further demonstrated regarding the recognition of complexity of dynamical system. The possible reason for the abrupt change could be the increasingly apparent influence of human beings upon the climate and environment since the late 1970s and the early 1980s. But no obvious abrupt changes have been detected in these three regions of the middle and lower reaches of the Yangtze River, south China, and southwest
China, which indicates that the complexity of precipitation in those regions is the outcome of the interaction of multiple factors. The human factor is only one of the important elements among them, which work together to influence those regions.

By a further analysis of the annual RR of the three regions of the middle and lower reaches of the Yangtze River, south China, and southwest China, it is found that the annual RR in those regions is apparently smaller than that in the other 


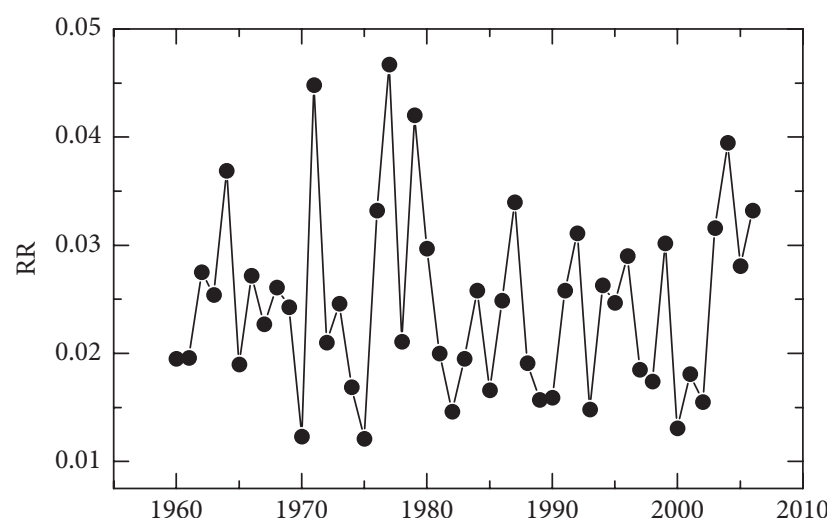

(a)

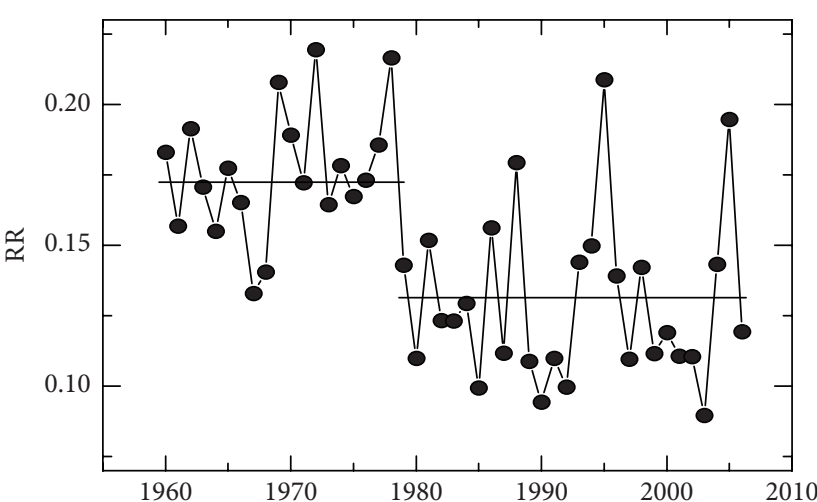

(c)

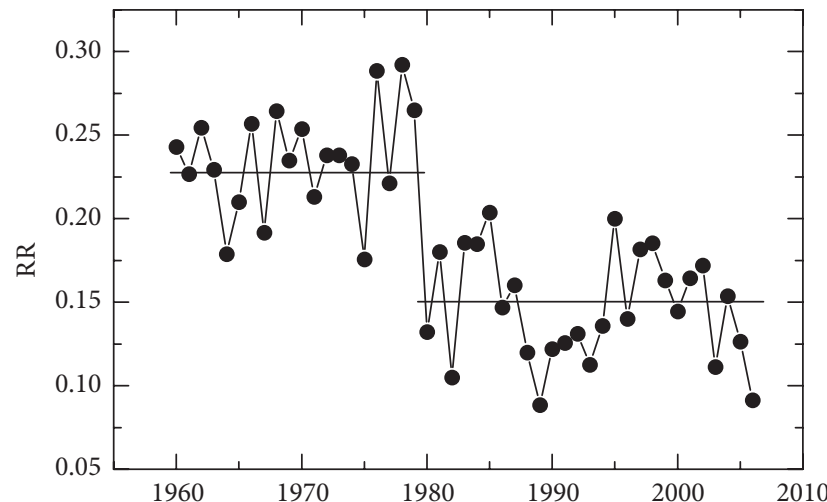

(e)

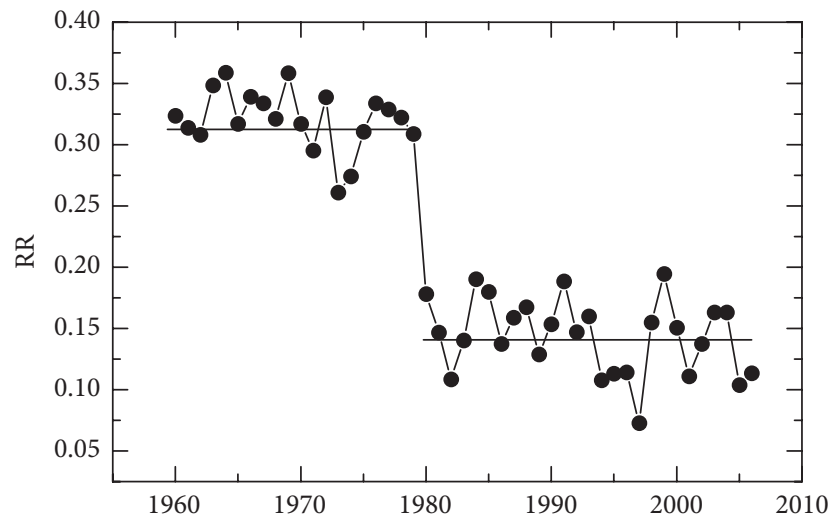

(g)

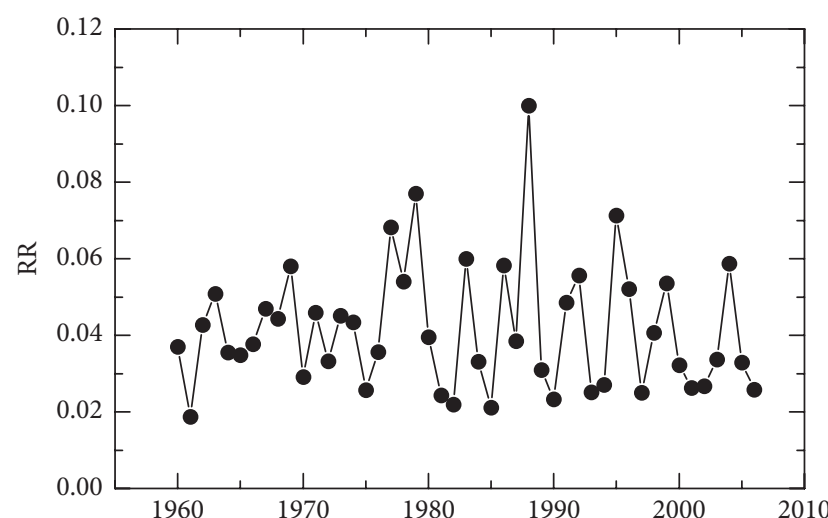

(b)

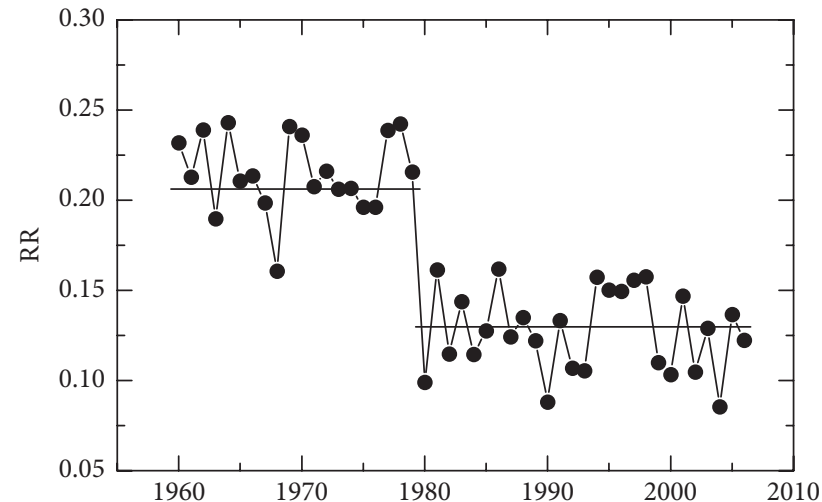

(d)

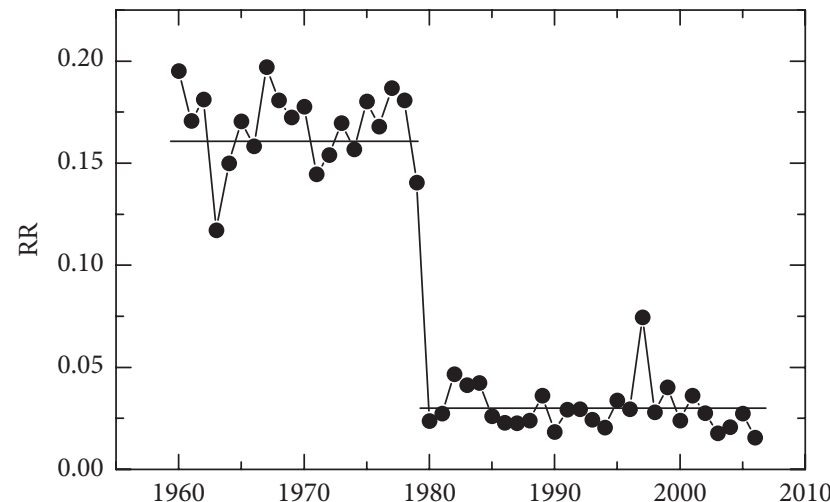

(f)

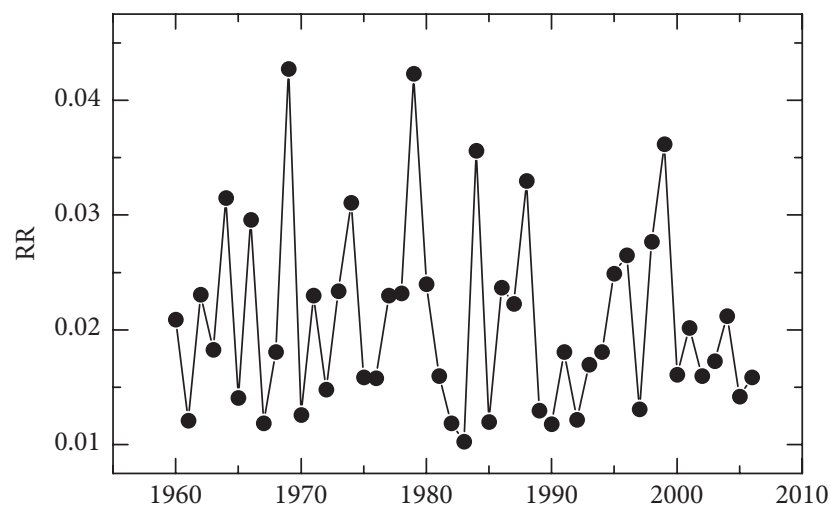

(h)

FIGURE 4: The charts of annual changes of precipitation RR of each region in China. 
five regions. It indicates the complexity is higher in those three regions, which is in agreement with the comparatively low accuracy of the precipitation forecast in those regions over past years. The main cause of the situation is because the climate system corresponding to the above regions is the result of cowork of various equivalent factors. It provides a feasible thought of data weather report. For the regions of comparatively complex dynamic structure, the predictability can be improved by attempts to increase perturbations. For the regions of comparatively simple dynamic structure, the calculation could be simplified by attempts to reduce certain microperturbations.

\section{Conclusion and Discussion}

In the paper the quantitative RR analysis is applied to the research of insect amount model, and the founding indicates the quantitative RR analysis can effectively recognize the changes of dynamic structure. Based on it, this method can be applied to the analysis of daily precipitation materials of stations in China. The result demonstrates that this method can comparatively well recognize the complexity of the climate system corresponding to precipitation in China and further discovers abrupt changes took place in the late 1970s and the early 1980s, in agreement with years previously commonly recognized; therefore its effectiveness is further demonstrated regarding the recognition of complexity of dynamical system.

\section{Competing Interests}

The authors declare that they have no competing interests.

\section{Acknowledgments}

This study was supported by the National Significant Scientific Research Plan of Global Change Research (Approval nos. 2012CB955902 and 2013CB430204) and the National Natural Science Foundation (Approval nos. 41275074 and 41475073).

\section{References}

[1] Z.-B. Fu, "Studies on the observed abrupt climatic change," Scientia Atmospherica Sinica, vol. 18, pp. 373-384, 1994.

[2] H. B. Mann, "Nonparametric tests against trend," Econometrica, vol. 13, pp. 245-259, 1945.

[3] M. G. Kendall and G. Charles, Rank Correlation Methods, Griffin, London, UK, 1975.

[4] R. Yamamoto, T. Iwashima, and N. K. Sanga, "Climatic jump, a hypothesis in climate diagnosis," Journal of the Meteorology Society Japan, vol. 63, pp. 1157-1160, 1985.

[5] W.-J. Dong and J.-P. Li, "Abrupt climate change detection based on heuristic segmentation algorithm," Acta Physica Sinica, vol. 54, pp. 5494-5499, 2005.

[6] Z.-Q. Gong, G.-L. Feng, S.-Q. Wan, and J.-P. Li, "Analysis of features of climate change of Huabei area and the global climate change based on heuristic segmentation algorithm," Acta Physica Sinica, vol. 55, no. 1, pp. 477-484, 2006.
[7] G.-L. Feng, W. Hou, and W.-J. Dong, "Technique for distinguishing dynamical species in the temperature time series of Yangtze River delta," Acta Physica Sinica, vol. 55, no. 2, pp. 962968, 2006.

[8] R. Zhi, Y. Lian, and G.-L. Feng, “The influence of different scale systems on precipitation analyzed on the basis of power-law exponent," Acta Physica Sinica, vol. 56, no. 3, pp. 1837-1842, 2007.

[9] W.-P. He, T. He, H.-Y. Cheng, W. Zhang, and Q. Wu, "A new method to detect abrupt change based on approximate entropy," Acta Physica Sinica, vol. 60, no. 4, Article ID 049202, 2011.

[10] V. N. Livina, S. Havlin, and A. Bunde, "Memory in the occurrence of earthquakes," Physical Review Letters, vol. 95, no. 20, Article ID 208501, 2005.

[11] W.-P. He, G.-L. Feng, Q. Wu, S.-Q. Wan, and J.-F. Chou, "A new method for abrupt change detection in dynamic structures," Nonlinear Processes in Geophysics, vol. 15, no. 4, pp. 601-606, 2008.

[12] G.-Q. Sun, S.-L. Wang, R. Qian, J. Zhen, and Y.-P. Wu, "Effects of time delay and space on herbivore dynamics: linking inducible defenses of plants to herbivore outbreak," Scientific Reports, vol. 5, Article ID 11246, 2015.

[13] G.-Q. Sun, Z.-Y. Wu, Z. Wang, and Z. Jin, "Influence of isolation degree of spatial patterns on persistence of populations," Nonlinear Dynamics, vol. 83, no. 1-2, pp. 811-819, 2016.

[14] J. P. Eckmann, S. O. Kamphorst, and D. Ruelle, "Recurrence plots of dynamical systems," Europhysics Letters, vol. 54, pp. 973-977, 1987.

[15] C. L. Webber Jr. and J. P. Zbilut, "Dynamical assessment of physiological systems and states using recurrence plot strategies," Journal of Applied Physiology, vol. 76, no. 2, pp. 965-973, 1994.

[16] J. P. Zbilut, A. Giuliani, and C. L. Webber Jr., "Detecting deterministic signals in exceptionally noisy environments using cross-recurrence quantification," Physics Letters, Section A: General, Atomic and Solid State Physics, vol. 246, no. 1-2, pp.122128, 1998.

[17] J. P. Zbilut and C. L. Webber Jr., "Embeddings and delays as derived from quantification of recurrence plots," Physics Letter A, vol. 171, no. 3-4, pp. 199-203, 1992.

[18] L. L. Trulla, A. Giuliani, J. P. Zbilut, and J. Webber, "Recurrence quantification analysis of the logistic equation with transients," Physics Letters A, vol. 223, no. 4, pp. 255-260, 1996.

[19] N. Marwan, M. C. Romano, M. Thiel, and J. Kurths, "Recurrence plots for the analysis of complex systems," Physics Reports, vol. 438, no. 5-6, pp. 237-329, 2007.

[20] D. B. Vasconcelos, S. R. Lopes, R. L. Viana, and J. Kurths, "Spatial recurrence plots," Physical Review E, vol. 73, no. 5, Article ID 056207, 10 pages, 2006.

[21] X.-M. Guo, C.-P. Li, and D.-L. Lu, "Application of recurrence quantification analysis and approximate Entropy in classification and recognition of heart sound," Journal of Data Acquisition and Processing, vol. 28, no. 5, pp. 559-564, 2013.

[22] F. Dong, N.-D. Jin, and Y. Wang, "Multi-scale recurrence quantification analysis of the dynamic characteristics of two phase flow pattern," Acta Physica Sinica, vol. 57, pp. 6145-6154, 2008.

[23] J. Yang, B.-Y. Zhou, Y. Zhang, and Y. Zhou, "Compressor fault diagnosis based on recurrence plot analysis," Computer \& Digital Engineering, vol. 41, no. 6, pp. 984-986, 2013. 


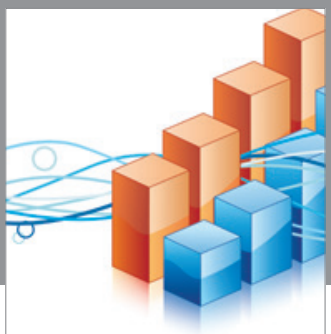

Advances in

Operations Research

vatem alat4

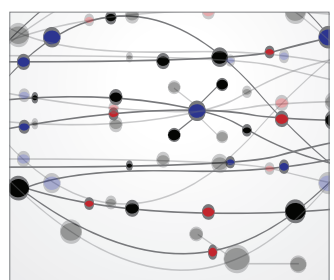

\section{The Scientific} World Journal
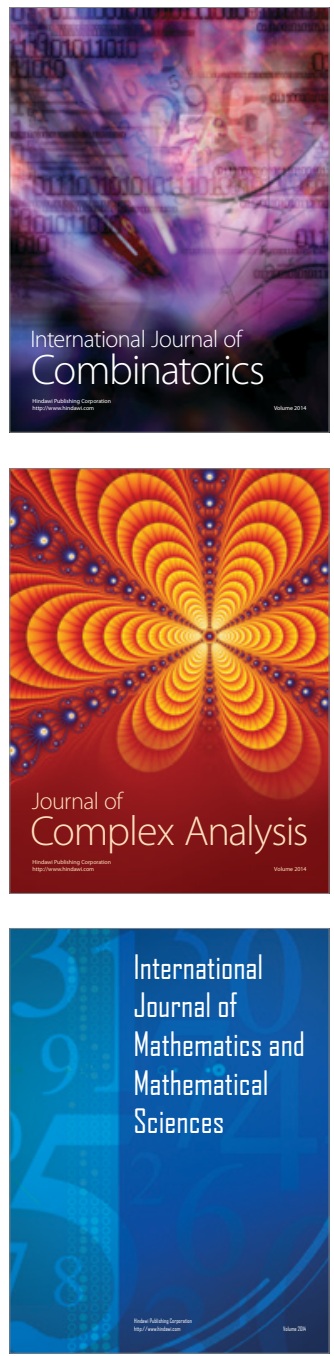
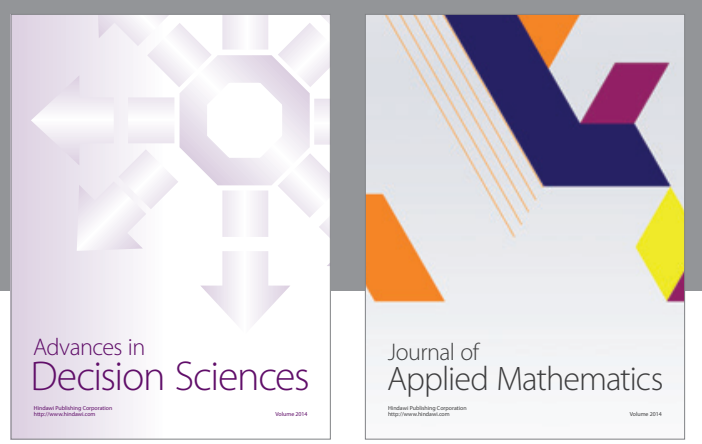

Algebra

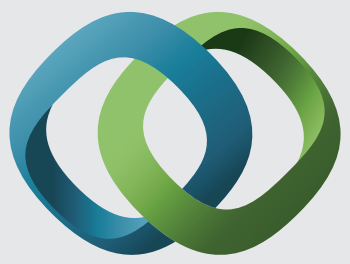

\section{Hindawi}

Submit your manuscripts at

http://www.hindawi.com
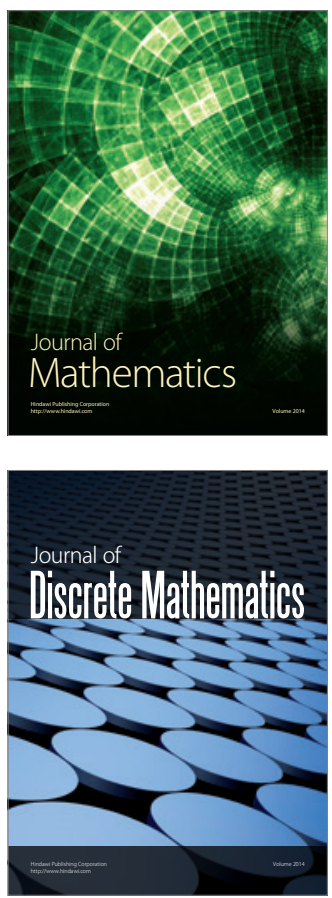

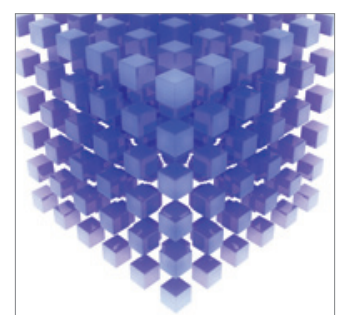

Mathematical Problems in Engineering
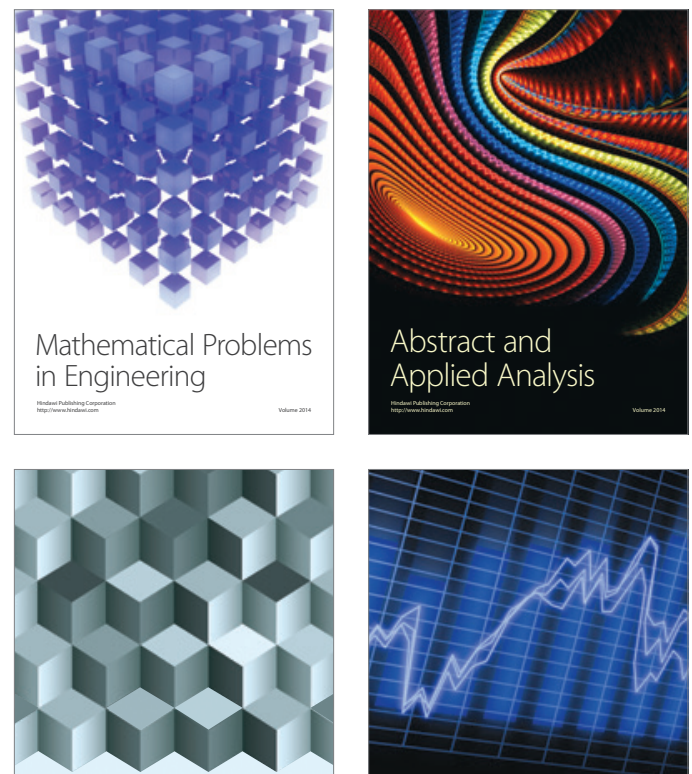

Journal of

Function Spaces

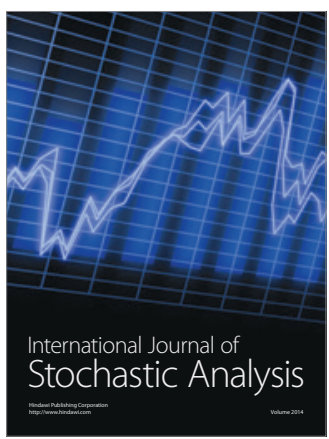

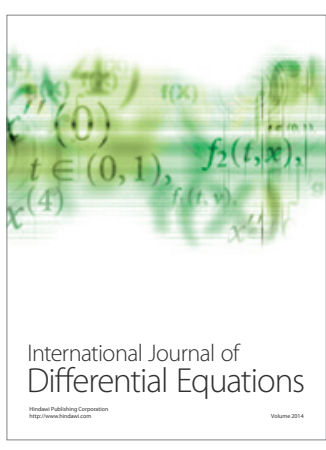
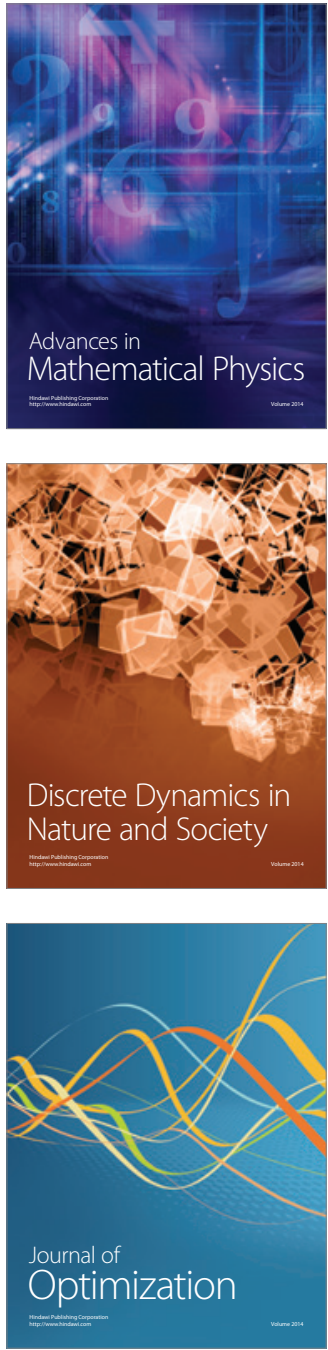